\title{
SAR Moving Objects Trajectory Estimation: Solving the Blind Angle Ambiguity with a Single Sensor *
}

\author{
Paulo A. C. Marques ${ }^{+,+}$, José M. B. Dias ${ }^{++}$ \\ ${ }^{+}$Instituto Superior de Engenharia de Lisboa \\ Departamento de Engenharia Electrotécnica e das Comunicações \\ R. Conselheiro Emídio Navarro 1, 1949-014 Lisboa Portugal \\ Fax: +351.21.831 71 14, E-mail: pmarques@isel.pt \\ ${ }^{++}$Instituto Superior Técnico - Instituto de Telecomunicações \\ Torre Norte, Piso 10, Av. Rovisco Pais, 1049-001 Lisboa \\ Fax: +351.21 .8418472$, E-mail: bioucas@lx.it.pt
}

\begin{abstract}
The Fisher information matrix for the unknown moving targets velocity parameters is computed as function of the antenna radiation pattern. The resultant matrix is analysed and the characteristics that the antenna gain should exhibit in order to enhance the estimation of the unknown moving objects velocity vector are deduced. Simulation results comparing the various antenna radiation patterns envisioning the velocity vector estimation using a single SAR sensor are presented.
\end{abstract}

\section{INTRODUCTION}

Detection, imaging, and trajectory parameter estimation of moving targets in SAR data is an active area of research. Several methods have been proposed to detect, image and estimate moving targets trajectory parameters in recent literature [1], [2], [3], [4], [5], [6]. Due to the so-called blind angle ambiguity, it is usually assumed that the target direction cannot be determined unless stereo measures are available [2].

In previous work [7] we showed that the returned echo from a moving target, in the slow-time frequency domain, is a scaled and shifted replica of the antenna radiation pattern, immersed in noise. The scale is proportional to the target cross-range velocity, while the shift depends on the target range speed. A Bayesian approach was adopted to derive an optimal estimator of the velocity parameters using a single SAR sensor. In this work we determine the Fisher information matrix for the unknown moving targets trajectory parameters as function of the antenna radiation pattern. The goal is to infer the characteristics

*This work was supported by Portuguese PRAXIS XXI program,under project 2/2.1.TIT/1580/95. that the antenna radiation pattern shape should have in order to enhance the moving targets velocity estimation ability, that is, to minimize the Cramer-Rao Lower Bound (CRLB) of each velocity parameter.

This paper is organized as follows: The next section establishes the signal model and notation. In the third Section the information matrix for the unknown moving targets velocity parameters is computed as function of the antenna radiation pattern. Section 4 presents some encouraging simulation results comparing the estimation enhancements with a single SAR sensor when the used antenna gains exhibit the characteristics inferred by the information matrix analysis.

\section{SIGNAL MODEL}

Consider the SAR geometry sketched in Fig. 1. The radar platform travels at speed $v_{r}$ in the navigation direction and has an antenna radiation pattern $a$ which is function of the observation angle. Consider a single moving target with constant complex reflectivity $f_{m}$, coordinates $\left(x_{m}, y_{m}\right)$ when the platform is at position $y=0$, and speed vector $\left(v_{x}, v_{y}\right)=\left(\mu_{m} v_{r}, b_{m} v_{r}\right)$ defined in the slant-plane $(x, y)$. Vector $\left(\mu_{m}, b_{m}\right)$ denotes the target relative velocity with respect to the radar. The ground $n^{t h}$ static target has complex reflectivity $f_{n}$ and coordinates $\left(x_{n}, y_{n}\right)$. When the radar is positioned at the coordinate $y=u$, the corresponding received signal in the slow-time frequency domain $k_{u}$ and the fast-time frequency domain $\omega$, after quadrature demodulation, is (see [7] for details)

$$
\begin{aligned}
S\left(k_{u}, \omega\right)= & P(\omega) A\left(k_{u}, \boldsymbol{\theta}\right) f_{m} e^{-j \sqrt{4 k^{2}-\left(\frac{k_{u}}{\alpha_{m}}\right)^{2}} X_{m}} e^{-j \frac{k_{u}}{\alpha_{m}} Y_{m}} \\
& +P(\omega) A\left(k_{u}, \boldsymbol{\theta}_{0}\right) \sum_{n} f_{n} e^{-j \sqrt{4 k^{2}-k_{u}^{2}} X_{n}} e^{-j k_{u} Y_{n}}
\end{aligned}
$$




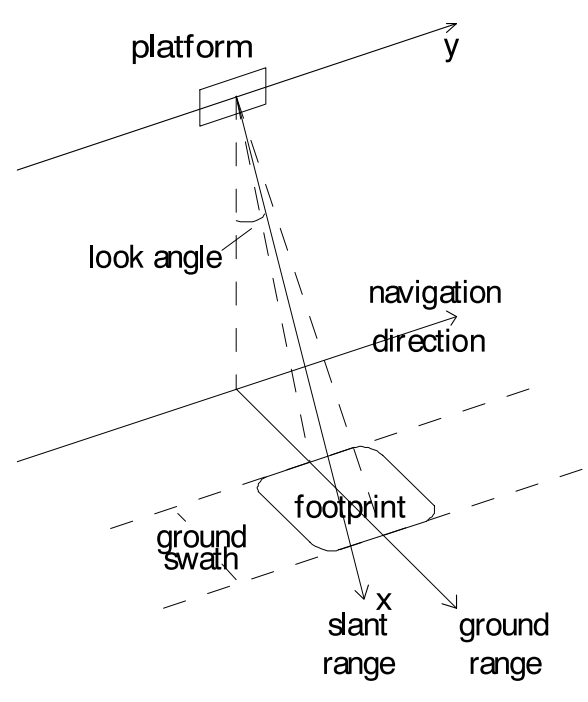

Figure 1: SAR geometry.

where $P(\omega)$ is the emitted chirp, $k=2 \pi / \omega, \boldsymbol{\theta}=\left(\mu_{m}, \nu_{m}\right)$, $A\left(k_{u}, \boldsymbol{\theta}\right) \propto a\left[1 /\left(2 \nu_{m}\right)\left(k_{u}-2 k \mu_{m}\right)\right]$, and as defined in [2] and [1]: $\nu_{m}=1+b_{m}, \alpha_{m} \equiv \sqrt{\mu_{m}^{2}+\nu_{m}^{2}}, \alpha_{m} Y_{m}=\mu_{m} x_{m}+$ $\nu_{m} y_{m}, \sqrt{X_{m}^{2}+Y_{m}^{2}}=\sqrt{x_{m}^{2}+y_{m}^{2}}$.

The first term on the right hand side of equation 1 is due to the moving target and the remaining terms are due to the static ground. The reflectivity of all targets has been taken to be independent of the aspect angle. Vector $\boldsymbol{\theta}_{0} \equiv(0,1)$ is the velocity vector of a static target. After pulse compression and phase compensation for the term due to the moving target (assuming $\alpha_{m}$ and $Y_{m}$ known), in the fast-time spatial domain, the received echo is

$$
\begin{array}{r}
S_{c}\left(k_{u}, X\right)=R_{p}\left(X-X_{m}\right) A\left(k_{u}, \boldsymbol{\theta}\right) f_{m}+A\left(k_{u}, \boldsymbol{\theta}_{0}\right) \times \\
\sum_{n} f_{n} R_{p}\left(X-X_{n}\right) e^{-j \frac{k_{u}^{2}}{4 k}\left(X_{n}+\frac{X_{m}}{\alpha_{m}^{2}}\right)} e^{-j k_{u}\left(Y_{n}-\frac{Y_{m}}{\alpha_{m}}\right)},
\end{array}
$$

where the Fresnel approximation has been taken, i.e. $\left[4 k^{2}-\left(k_{u} / \alpha_{m}\right)^{2}\right]^{1 / 2} \approx 2 k-\frac{1}{4 k}\left(k_{u} / \alpha_{m}\right)^{2} . \quad R_{p}(X)$ is the auto-correlation of the emitted chirp. Define $\mathbf{S}=$ $\left[S_{-N} \cdots S_{0} \cdots S_{N}\right]^{T}$, where $S_{i} \equiv S_{c}\left(k_{u_{i}}, X_{m}\right), k_{u_{i}}=$ $\frac{i}{2 N} \Delta K$, for $i=-N, \ldots, 0, \ldots, N$, where $\Delta K$ is the synthetic aperture bandwidth. Define also $\mathbf{A}(\boldsymbol{\theta})=$ $\left[A_{-N}(\boldsymbol{\theta}) \cdots A_{0}(\boldsymbol{\theta}) \cdots A_{N}(\boldsymbol{\theta})\right]$ where $A_{i}(\boldsymbol{\theta})=A\left(k_{u_{i}}, \boldsymbol{\theta}\right)$.

Let us assume for a while that the parameter $\boldsymbol{\theta}$ is known. In this case only the noise term in the expression (2) is random. Assuming that the number of static scatterers is large, none is predominant, and that they are uniformly distributed within a wavelength, then the noise is Gaussian distributed. Therefore vector $\mathbf{S}$ density conditioned to $\boldsymbol{\theta}$ and $f_{m}$ is

$$
p\left(\mathbf{S} \mid f_{m}, \boldsymbol{\theta}\right)=N\left(\widetilde{\mu}_{s}, \mathbf{C}_{s}\right),
$$

where $\widetilde{\mu}_{S}=E[\mathbf{S}]$ and $\mathbf{C}_{s}=E\left[\left(\mathbf{S}-\mathbf{m}_{s}\right)\left(\mathbf{S}-\mathbf{m}_{s}\right)^{H}\right]$.

In work [8] we show that $E[\mathbf{S}]=f_{m} \mathbf{A}(\boldsymbol{\theta})$ and $\mathbf{C}_{s}=$ $\frac{P L E_{p}}{\Delta X \Delta Y} \operatorname{diag}\left(\left|\left[A\left(\boldsymbol{\theta}_{0}\right)\right]_{i}\right|^{2}\right)$, for $i=-N, \ldots, N$, were $\mathrm{P}$ is the average power of the clutter; $\mathrm{L}$ is the cross-range length; $E_{p}$ is the energy of the transmitted pulse in range; $\Delta X$ and $\Delta Y$ are the resolution lengths in range and crossrange, respectively.

\section{ANTENNA RADIATION PATTERN SHAPE ENVISIONING MOVING TARGETS VELOCITY VECTOR ESTIMATION}

In this section we will compute the Fisher information matrix elements as function of the antenna radiation pattern shape. The goal is to infer the characteristics that the radiation pattern should exhibit in order to reduce the CRLB for the velocity parameters of the moving target.

\section{Information Matrix Computation}

The CRLB for a complex Gaussian PDF results in the Fisher information matrix:

$$
\begin{aligned}
{[I(\boldsymbol{\theta})]_{i j}=} & \operatorname{tr}\left[C_{s}^{-1}(\boldsymbol{\theta}) \frac{\delta C_{s}(\boldsymbol{\theta})}{\delta \theta_{i}} C_{s}^{-1}(\boldsymbol{\theta}) \frac{\delta C_{s}(\boldsymbol{\theta})}{\delta \theta_{j}}\right] \\
+ & 2 \operatorname{Re}\left[\frac{\delta \tilde{\mu}^{H}(\boldsymbol{\theta})}{\delta \theta_{i}} C_{s}^{-1}(\boldsymbol{\theta}) \frac{\delta \tilde{\mu}(\boldsymbol{\theta})}{\delta \theta_{j}}\right], \\
& i, j=1,2 .
\end{aligned}
$$

The first term in eq. (4) is null because the noise covariance matrix is independent of the moving target parameters.

Recalling that $\tilde{\mu}(\boldsymbol{\theta})=f_{m} \mathbf{A}(\boldsymbol{\theta})$,

$$
\frac{\delta \tilde{\mu}^{H}(\boldsymbol{\theta})}{\delta \mu_{m}}=f_{m}^{*} \frac{-k}{\nu_{m}}\left[\dot{A}_{0}(\boldsymbol{\theta}) \dot{A}_{1}(\boldsymbol{\theta}) \cdots \dot{A}_{N-1}(\boldsymbol{\theta})\right]
$$

$$
\frac{\delta \tilde{\mu}^{H}(\boldsymbol{\theta})}{\delta \nu_{m}}=f_{m}^{*} \frac{2 k \mu_{m}-k_{u}}{2 \nu_{m}^{2}}\left[\dot{A}_{0}(\boldsymbol{\theta}) \dot{A}_{1}(\boldsymbol{\theta}) \cdots \dot{A}_{N-1}(\boldsymbol{\theta})\right]
$$

The Fisher matrix elements are thus written:

$$
\begin{gathered}
I_{11}(\boldsymbol{\theta})=2 \frac{\left|f_{m}\right|^{2} \frac{k^{2}}{\nu_{m}^{2}}}{\frac{P L}{\Delta X Y Y}} \sum_{i=0}^{N-1} \frac{\left|\dot{A}_{i}(\boldsymbol{\theta})\right|^{2}}{\left|A\left(\boldsymbol{\theta}_{0}\right)_{i}\right|^{2}} \\
I_{22}(\boldsymbol{\theta})=2 \frac{\left|f_{m}\right|^{2}}{\frac{P L}{\Delta X \Delta Y}} \sum_{i=0}^{N-1} \frac{\left|\dot{A}_{i}(\boldsymbol{\theta})\right|^{2}}{\left|A\left(\boldsymbol{\theta}_{0}\right)_{i}\right|^{2}}\left(\frac{2 k \mu_{m}-k_{u i}}{2 \nu_{m}^{2}}\right)^{2}, \\
I_{12}(\boldsymbol{\theta})=I_{21}(\boldsymbol{\theta})=2 \frac{\left|f_{m}\right|^{2} \frac{-k}{\nu_{m}}}{\frac{P L}{\Delta X \Delta Y}} \sum_{i=0}^{N-1} \frac{|\dot{A}(\boldsymbol{\theta})|^{2}}{\left|A\left(\boldsymbol{\theta}_{0}\right)_{i}\right|^{2}} \frac{2 k \mu_{m}-k_{u i}}{2 \nu_{m}^{2}} .
\end{gathered}
$$


The element $I_{11}$ refers to the slant-range speed, whereas $I_{22}$ refers to the cross-range velocity. A brief analysis of this two elements enables us to do the following statements:

- The worst situation occurs when there is complete superposition. In that case, the first derivative of the antenna radiation pattern should exhibit significant values on the frequency values where the antenna radiation pattern decays towards zero.

- For small displacements, the range velocity component is easier to estimate than the cross-range component since the information matrix element due to it has approximately $\nu_{m} / \mu_{m}$ times the value of its counterpart.

- The element which refers to the azimuth velocity is affected by a quadratic factor centered on the central frequency of the radiation pattern. This factor enhances the first derivative by a factor proportional to its distance from the central frequency, suggesting that the radiation pattern should have significant variation on its extreme frequencies.

Based on this results one expects that a Gaussian antenna radiation pattern will provide worst CRLB than a triangular one. A trapezoidal shape should exhibit better results than the other two, since it has higher derivative values at it's extremes.

\section{ANTENNA RADIATION PATTERN SHAPE EVALUATION}

In this section the suggestions due to the information matrix analysis will be applied to compare antenna radiation pattern shapes envisioning the detection of the complete moving objects velocity vector. The following antenna radiation pattern shapes will be compared: Gaussian, triangular and trapezoidal. The simulation parameters are those described in table 1.

Figures 2 and 3 plot the CRLB square root for the range speed and cross-range speed for the several considered shapes versus the ground superposition. The label trapezoid $(x)$ refers to a trapezoid with a length in the top which is $1-x$ the length of its base.

As expected, the trapezoidal shape performs better than all the others. It is followed by the triangular function. Notice that the Gaussian shape is near one order of magnitude worst than the other considered antenna radiation patterns .

In Figures 4 and 5 the theoretical evaluation is corroborated by simulation experiments using the estimator developed in [7]. This simulation confirms the advantages
Table 1: Simulation parameters.

\begin{tabular}{|c|c|}
\hline Parameter & Value \\
\hline Carrier frequency & $5 \mathrm{GHz}$ \\
Altitude & $10 \mathrm{Km}$ \\
Velocity & $300 \mathrm{Km} / \mathrm{h}$ \\
Look angle & $20^{\circ}$ \\
SNR & $0 \mathrm{~dB}$ \\
\hline
\end{tabular}

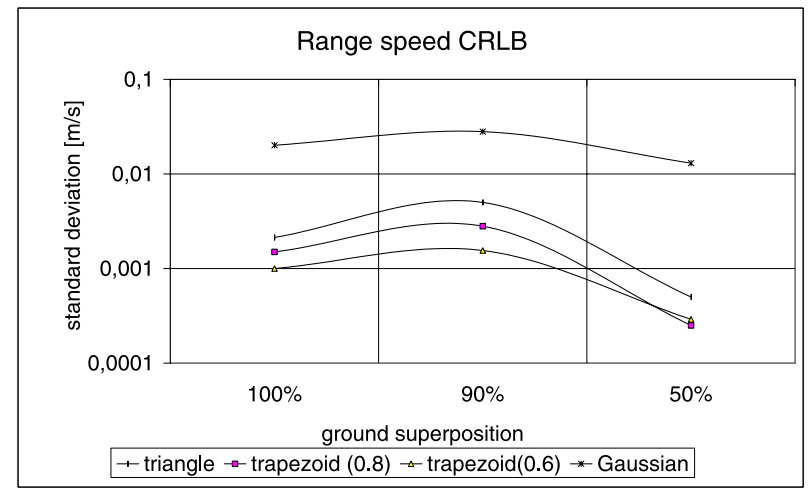

Figure 2: CRLB of range speed parameter versus groundreturn superposition.

of using antenna radiation patterns which exhibit high derivative values at its extremes.

\section{CONCLUDING REMARKS}

The Fisher information matrix for the unknown moving targets velocity parameters has been determined as function of the antenna radiation pattern. This enabled us to infer that the antenna radiation pattern should exhibit significant variation on its extreme frequencies in order to enhance the velocity estimation ability using a single SAR sensor.

Simulation results which corroborate the theoretical evaluation were presented.

\section{References}

[1] Mehrdad Soumekh, Synthetic Aperture Radar Signal Processing with MATLAB algorithms, WILEYINTERSCIENCE, 1999.

[2] Mehrdad Soumekh, "Reconnaissance with ultra wideband uhf synthetic aperture radar", IEEE Signal Processing Magazine, vol., pp. 21-40, July 1995. 


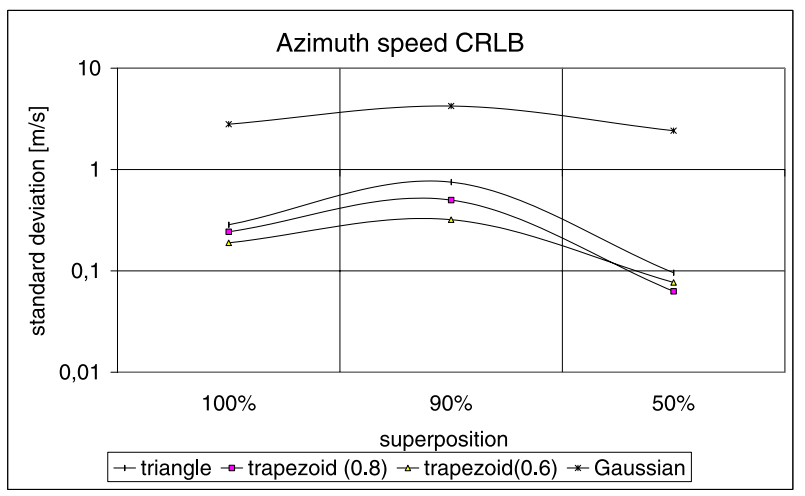

Figure 3: CRLB of cross-range speed parameter versus illumination shape.

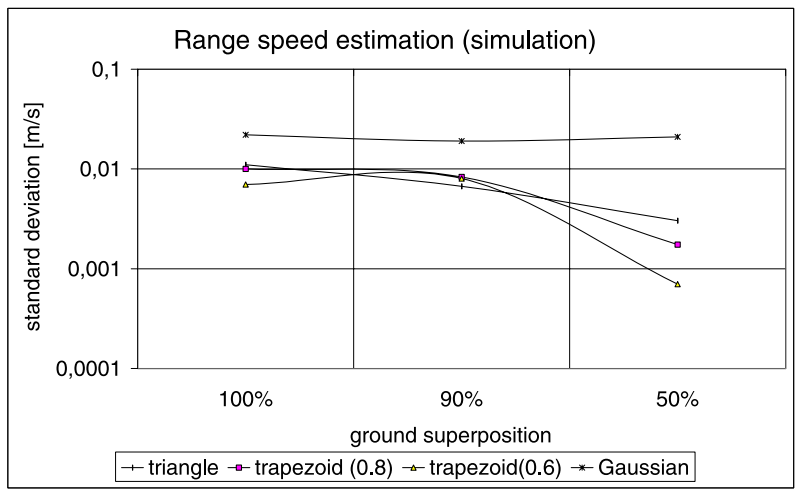

Figure 4: Standard deviation of range speed parameter estimation versus ground-return superposition.

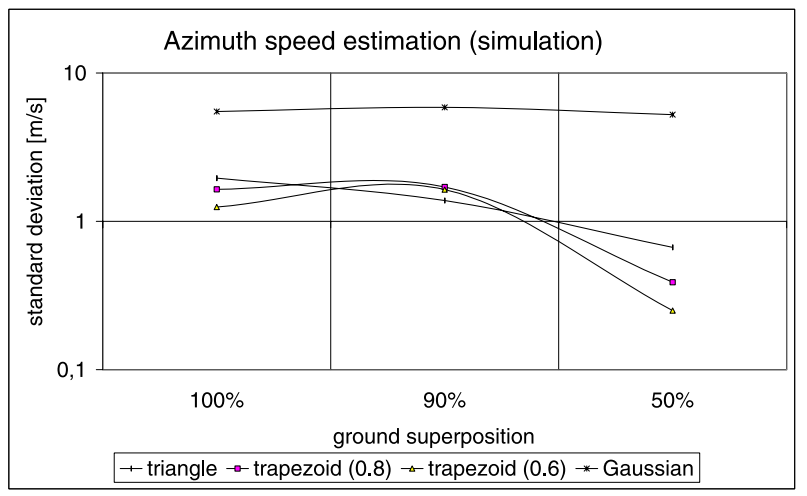

Figure 5: Standard deviation of cross-range speed parameter estimation versus ground-return superposition.
[3] S. Barbarossa, "Detection and imaging of moving objects with synthetic aperture radar", IEE Proceedings$F$, vol. 139, pp. 79-88, February 1992.

[4] Paulo Marques; José Dias, "Efficient detection and ground mapping of selected moving targets using sar raw-data", in Proc. of the IGARSS'99, 1999.

[5] Martin Kirsht, "Detection, velocity estimation and imaging of moving targets with single-channel sar", in Proc. of the EUSAR'98, pp. 587-590, 1998.

[6] Richard P. Perry; Robert C DiPietro; Ronald L. Fante, "Sar detection and imaging of moving targets", in Proc. of the EUSAR'98, pp. 579-582, 1998.

[7] Paulo Marques and José Dias, "Optimal detection and imaging of moving objects with unknown velocity", in Proc. of the 3rd European Conference on Synthetic Aperture Radar, 2000.

[8] Paulo Marques and José Dias, "Blind angle ambiguity resolution in parameter estimation of moving targets using a single sar sensor", in Proc. of the EUSIPCO'2000 (to be published), 2000. 